\title{
Synergistic effect of metformin and medroxyprogesterone 17-acetate on the development of endometrial cancer
}

\author{
NAN MU ${ }^{1 *}$, MEI DONG ${ }^{2 *}$, LEI LI $^{1 *}$, MIN XIA $^{1}$, LUYUN QV $^{1}$, YINGMEI WANG ${ }^{3}$, \\ CHANGYAN DONG $^{1}$, YONGHUA CHEN ${ }^{1}$, YING ZUO ${ }^{1}$, JIANQING HOU ${ }^{1}$ and FENGXIA XUE ${ }^{3}$ \\ Departments of ${ }^{1}$ Gynecology and Obstetrics and ${ }^{2}$ Cardiology, Yantai Yuhuangding Hospital, \\ Affiliated to Qingdao University, Yantai, Shandong 264000; ${ }^{3}$ Department of Gynecology and Obstetrics, \\ Tianjin Medical University General Hospital, Tianjin 300052, P.R. China
}

Received September 4, 2017; Accepted January 23, 2018

DOI: $10.3892 /$ or.2018.6236

\begin{abstract}
Accumulating data indicate that insulin resistance and unopposed estrogen are important risk factors of endometrial cancer (EC). Medroxyprogesterone 17-acetate (MPA) has been used in the treatment of EC for many years. However, the therapeutic effect of this agent on EC has not been satisfactory. 36 arMetformin was recently reported to be a promising agent for the treatment of malignant diseases including EC. However, information on the synergistic effect of the two agents in EC is limited. With the aim to evaluate the synergistic effect of metformin and MPA, we conducted the present study in vitro and in vivo. We found that the combined application of metformin and MPA significantly inhibited the proliferation of the Ishikawa cells and arrested the cells in the G0/G1 phase. Furthermore, the apoptosis rate of the Ishikawa cells was significantly increased. In the animal study, the development of the xenograft tumors was significantly suppressed by the combined application of the two agents. Further investigation revealed that the synergistic inhibitory effect of the two agents on EC can be at least partly, explained by the decreased expression of cyclin D1 and cyclin $\mathrm{E}$. The results of the current study provide novel insights into the treatment of EC.
\end{abstract}

Correspondence to: Professor Jianqing Hou, Department of Gynecology and Obstetrics, Yantai Yuhuangding Hospital, Affiliated to Qingdao University, 20 Yuhuangding East Road, Zhifu, Yantai, Shandong 264000, P.R. China

E-mail: prohoujianqing@outlook.com

Professor Fengxia Xue, Department of Gynecology and Obstetrics, Tianjin Medical University General Hospital, 154 Anshan Road, He Ping, Tianjin 300052, P.R. China

E-mail: proxuefengxia@outlook.com

${ }^{*}$ Contributed equally

Key words: endometrial cancer, metformin, medroxyprogesterone 17-acetate, synergistic effect, treatment

\section{Introduction}

Endometrial cancer (EC) is a common gynecological malignancy in developed countries. Unfortunately, the etiology of EC remains unclear. Traditionally, 'unopposed estrogen' is considered responsible for the carcinogenesis of EC $(1,2)$. According to this hypothesis, without enough progestin, estrogen urges the malignant transformation of the endometrium by stimulating the proliferation and inhibiting the apoptosis of endometrial cells. Research supporting this hypothesis has revealed that postmenopausal women receiving hormone replacement therapy (HRT) containing estrogen alone, face increased risk of developing EC, but, this risk decreased when progestin was added (3). However, 'unopposed estrogen' alone, cannot totally explain the pathogenesis of EC. Recently, accumulating evidence has revealed that insulin resistance is probably an important risk factor of EC $(4,5)$. In the state of insulin resistance, elevated levels of insulin stimulate the development of EC by activating phosphoinositide 3-kinase/protein kinase B (PI3K/PKB) and mitogen-activated protein kinase/extracellular regulated kinase (MAPK/ERK) signaling pathways $(6,7)$. Furthermore, insulin was found to be an independent risk factor of EC for both pre- and postmenopausal women $(8,9)$. Since estrogen and insulin are established risk factors for the development of EC, they appear to be targets for the treatment of EC.

To date, medical treatment for EC consists primarily of surgery, including total abdominal hysterectomy, bilateral salpingo-oophorectomy and additional lymph node dissection (10). However, the surgical operations add a potential threat to approximately $20 \%$ of patients with EC who are premenopausal early-phase patients wishing to maintain their fertility (11). In addition, for some patients with EC, surgical treatment appears to be unsuitable because of morbid obesity and other serious complications. Recently, several studies on the non-surgical treatment of EC have revealed new insights into this malignancy $(12,13)$. Progestin and metformin are promising candidates as therapeutic agents for EC treatment (14). Progestin has been employed in the treatment of EC for many years. However, the effect of progestin on the treatment of EC is poor (15). Metformin is a popular insulin-sensitizing agent used in the treatment of type II diabetes mellitus. Furthermore, 
the antitumor effects of metformin have attracted scientific attention (16). In addition, the results provided by some studies reported that metformin plays an important role in the treatment of several malignant diseases including EC (17).

However, there is limited knowledge about the synergistic effect of progestin and metformin on EC. In the present study, we examined the combined effects of progestin-MPA, a synthetic progestin- and metformin on EC in vitro and in vivo. Furthermore, the mechanism of the effect was also explored.

\section{Materials and methods}

Cell line and reagents. The well-differentiated human EC cell line Ishikawa (a kindly gift from Professor Fengxia Xue), expressing estrogen and progestin receptors was used in the present study. The cells were maintained in phenol red-free DMEM/F12 with $10 \%$ fetal bovine serum (FBS) at $37^{\circ} \mathrm{C}$ in $5 \% \mathrm{CO}_{2}$. We passaged the Ishikawa cells every 3 to 5 days. Metformin, medroxyprogesterone 17-acetate (MPA) and MTT dye were purchased from Sigma-Aldrich (St. Louis, MO, USA). Caspase-3 ELISA kit was purchased from Cell Signaling Technology (Beverly, MA, USA). All primary and secondary antibodies were purchased from Santa Cruz Biotechnology (Dallas, TX, USA): $\beta$-actin monoclonal antibody (sc-47778), cyclin D1 monoclonal antibody (sc-450), cyclin E monoclonal antibody (sc-247), phosphor-Akt monoclonal antibody (sc-514032), phosphor-ERK monoclonal antibody (sc-7383)

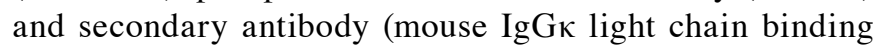
protein) (sc-516102).

Proliferation assay. The effects of metformin and (or) MPA treatment on Ishikawa cell proliferation were determined by an MTT assay. EC cells were plated and grown in 96-well plates at a concentration of about 8,000 cells $/ \mu 1$ for $24 \mathrm{~h}$. The EC cells were then treated with metformin and (or) MPA for $72 \mathrm{~h}$ at a concentration of 5 and $1 \mu \mathrm{M}$, respectively. The cells treated with phosphate-buffered saline (PBS) were considered as the control group. Cell densities at different time-points $(1,12,24,48$ and $72 \mathrm{~h})$ were determined by metabolic conversion of the MTT dye. We added MTT $(5 \mathrm{mg} / \mathrm{ml})$ to the $96-w e l l$ plates at $10 \mu \mathrm{l}$ in every well, and then incubated those plates for an additional hour. Finally, we ended the MTT reaction through the addition of $100 \mu \mathrm{l}$ of dimethyl sulfoxide (DMSO). Subsequently, the MTT assay results were examined by determining absorption at $595 \mathrm{~nm}$. The effect of metformin and (or) MPA on the proliferation of Ishikawa cells was assessed as a percentage of the control cell-growth obtained from the PBS-treated cells grown in the same 96-well plates. All experiments were performed in triplicate and repeated three times.

Apoptosis assay. Cells were cultured in 6-well plates at a concentration of $\sim 2 \times 10^{5}$ cells/well for $24 \mathrm{~h}$ and then treated with metformin $(5 \mu \mathrm{M})$ and (or) MPA $(1 \mu \mathrm{M})$ in $0.5 \%$ stripped serum for an additional $72 \mathrm{~h}$. Cells treated with PBS were considered as the control group. Caspase- 3 kit was used to determine the apoptosis rate of the Ishikawa cells at different time-points $(1,12,24,48$ and $72 \mathrm{~h})$. Reagents were added according to the manufacturer's instructions and the ELISA plate was examined by assessing absorption at $450 \mathrm{~nm}$.
All experiments were performed in triplicate and repeated three times.

Flow cytometry. In order to explore the mechanism of the effects of metformin and (or) MPA on Ishikawa cells, we examined the cell cycle profile. The cells were plated at a concentration of $2 \times 10^{5}$ cells/well in 6 -well plates for $24 \mathrm{~h}$. Subsequently, the cells were starved overnight and then, treated with $15 \%$ serum for $72 \mathrm{~h}$ with metformin $(5 \mu \mathrm{M})$ and (or) MPA $(1 \mu \mathrm{M})$. The cells were then collected and washed with PBS, fixed in a $90 \%$ methanol solution and stored at $-20^{\circ} \mathrm{C}$ until the flow cytometric analysis was performed. In the analysis, the Ishikawa cells were firstly washed and centrifuged with cold PBS, suspended in $100 \mu \mathrm{l}$ PBS and $10 \mu \mathrm{l}$ of RNase. Then the solution $(250 \mu \mathrm{g} / \mathrm{ml})$ was incubated at $37^{\circ} \mathrm{C}$ for $30 \mathrm{~min}$. After incubation, $110 \mu \mathrm{l}$ of propidium iodide (PI) stain $(100 \mu \mathrm{g} / \mathrm{ml})$ were added to each tube and incubated at $4^{\circ} \mathrm{C}$ for at least $30 \mathrm{~min}$ before the examination. Flow cytometric analysis was performed on a CyAn machine (Beckman Coulter, Inc., Miami, FL, USA). ModFit (Verity Software House, Topsham, ME, USA) was used for the analysis for dead cells and cell debris.

Animal study. Four-week old female Balb/C nude mice with a mean body mass of 15-18 g were purchased from Charles River Laboratories (Beijing, China). After one week to adapt to the new environment, the mice were injected subcutaneously into the right flank with $\sim 5 \times 10^{6}$ Ishikawa cells. One week after cell implantation, all the tumors became palpable. The mice were randomly divided into four groups $(n=8$ for each group). One group received no treatment and served as the control group. The other three groups were treated with metformin (250 mg/kg, daily, per os) and (or) MPA (1 mg in $0.1 \mathrm{ml}$ volume, weekly, intramuscular). Tumor dimensions were measured twice a week. Tumor volume was calculated using the following formula: $V=a \times b \times b / 2$ ( $a$ is the longest axis and $b$ the shortest axis of the tumor). On day 60, the animals were sacrificed. The tumors were excised and frozen for further analysis. All procedures involving animals in the present study were approved by the Animal Care and Use Committee of Yantai Yuhuangding Hospital Affiliated to Qingdao University. The welfare of the animals was well-ensured in the present study (18).

Western blot analysis. In the present study, the expression of cyclin D1, cyclin E and phosphorylated Akt and ERK was examined by western blot analysis. Frozen tumor tissues were thawed and lysed in RIPA buffer (1\% NP 40, 0.5 sodium deoxycholate and $0.1 \%$ SDS). Lysates (10 $\mu \mathrm{g}$ of protein) were separated by gel electrophoresis and transferred onto the nitrocellulose membranes. Subsequently, the membranes were blocked by $5 \%$ non-fat dry milk and $0.1 \%$ Tween-20 to saturate non-specific sites. The primary antibodies were diluted $(1: 1,000)$ and incubated overnight at $4^{\circ} \mathrm{C}$. The secondary antibody was diluted $(1: 4,000)$ and incubated at room temperature for $60 \mathrm{~min}$. The signals were detected using the ECL reagent (GE Healthcare, Chicago, IL, USA) and the ImageQuant LAS 4000 system (GE Healthcare). The gray value of each band in the imaging data was analyzed using Quantity One software (Bio-Rad Laboratories, Inc., Hercules, 
Table I. Gray values in the western blot analysis.

\begin{tabular}{lcccc}
\hline & \multicolumn{3}{c}{ Gray value } \\
\cline { 2 - 5 } Proteins & Control & Metformin & MPA & Metformin+MPA \\
\hline Cyclin D1 & $1.21 \pm 0.11$ & $0.88 \pm 0.08$ & $0.95 \pm 0.08$ & $0.55 \pm 0.10$ \\
Cyclin E & $0.98 \pm 0.12$ & $0.76 \pm 0.06$ & $0.80 \pm 0.07$ & $0.48 \pm 0.09$ \\
p-Akt & $0.90 \pm 0.08$ & $0.89 \pm 0.09$ & $0.88 \pm 0.08$ & $0.84 \pm 0.08$ \\
p-ERK & $0.81 \pm 0.07$ & $0.77 \pm 0.10$ & $0.80 \pm 0.09$ & $0.76 \pm 0.09$ \\
\hline
\end{tabular}

CA, USA). The ratios of the gray value of the target band and $\beta$-actin used in the analysis are listed in Table I.

Statistical analysis. The data were analyzed using SAS software package (version 9; SAS Institute, Cary, NC, USA). Significance of difference between the variables, except for flow-cytometry data, was examined by the Student's t test. Mann-Whitney U test was used to analyze the data of flow cytometry. All P-values were two-sided among which a value $<0.05$ was considered to indicate a statistically significant difference.

\section{Results}

Effect of metformin and (or) MPA on cell proliferation. The effect of metformin and (or) MPA on the proliferation of cancer cells was determined at different time-points in the present study. As displayed in Fig. 1, one hour after the treatment, there was no significant difference among the proliferation rate of the cells treated with PBS, metformin, MPA or metformin + MPA. At the time-points of 12, 24, 48 and $72 \mathrm{~h}$ after treatment, the proliferation rate of the cells treated with metformin (P-value $=0.047,0.041,0.026$ and 0.020 , respectively) MPA (P-value $=0.048,0.043,0.030$ and 0.022 , respectively) and metformin + MPA (P-value $=0.044,0.038$, 0.019 and 0.016 , respectively) was significantly lower than that of the cells treated with PBS. Furthermore, the proliferation inhibitory effect of metformin + MPA was found significantly stronger than that of metformin $(\mathrm{P}$-value $=0.045,0.042$ and 0.039 , respectively) or MPA (P-value $=0.040,0.037$ and 0.026 , respectively) used alone at 24,48 and $72 \mathrm{~h}$ after treatment.

Effect of metformin and (or) MPA on cell apoptosis. The apoptosis assay was performed by examining the activity of caspase- 3 which is a biomarker for cell apoptosis. As displayed in Fig. 2, twelve hours after treatment, the apoptosis rate of cells treated with metformin + MPA was significantly higher than that of the other three groups $(\mathrm{P}=0.013$ for the metformin group, 0.011 for the MPA group and 0.006 for the control group). At the time-points of 24,48 and $72 \mathrm{~h}$ after treatment, the apoptosis rate of the cells treated with metformin $(\mathrm{P}=0.041$, 0.037 and 0.033 , respectively), MPA $(\mathrm{P}=0.048,0.042$ and 0.038 , respectively $)$ and metformin + MPA $(\mathrm{P}=0.002,<0.001$ and $<0.001$, respectively) were all significantly higher than that of the cells treated with PBS. Furthermore, at 24, 48 and $72 \mathrm{~h}$ after treatment, the proliferation inhibitory effect of metformin + MPA was found significantly stronger than that

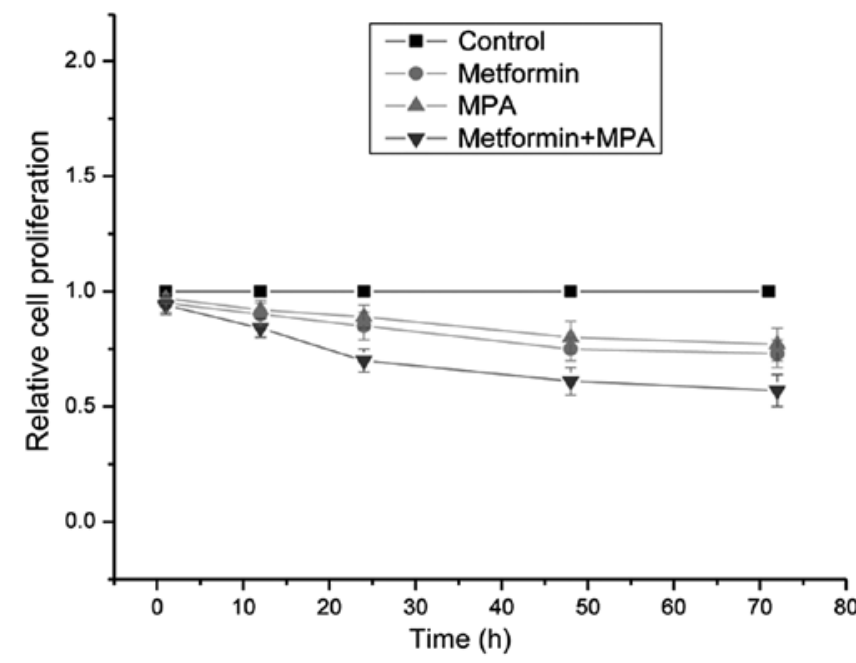

Figure 1. Effect of metformin and (or) MPA on the proliferation of Ishikawa cells. Metformin, MPA and metformin + MPA all inhibited the proliferation of Ishikawa cells in a time-dependent manner. The combined use of metformin and MPA exhibited significantly stronger inhibitory effect than either of the other agents used alone. The results are presented as the mean $\pm \mathrm{SE}$.

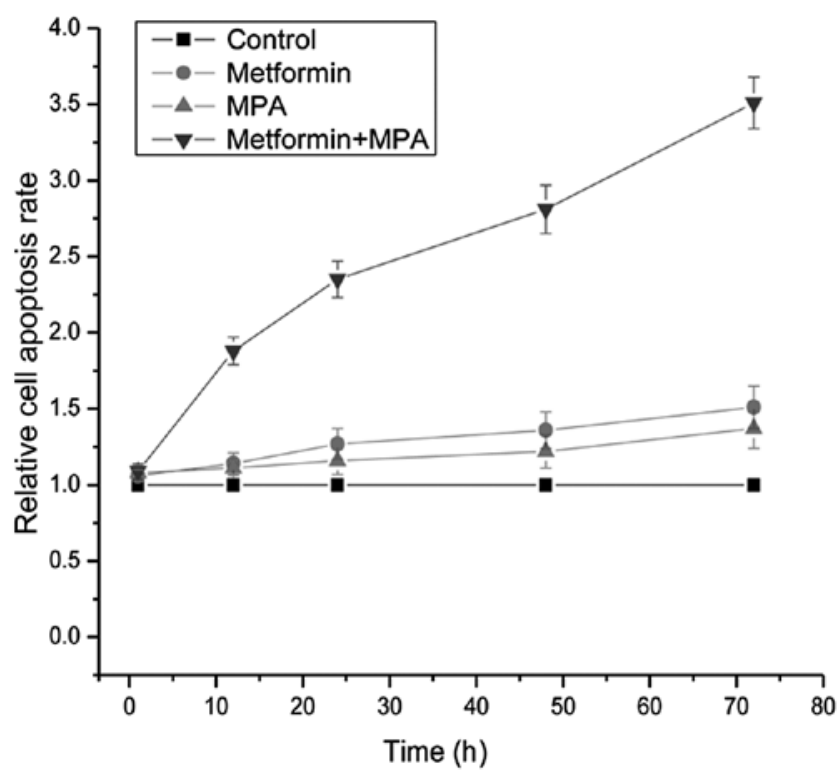

Figure 2. Effect of metformin and (or) MPA on the apoptosis of Ishikawa cells. Metformin, MPA and metformin + MPA all promoted the apoptosis of Ishikawa cells in a time-dependent manner. The combined use of metformin and MPA demonstrated significant stronger apoptosis-promoting effect than either of the agents used alone. The results are presented as mean $\pm \mathrm{SE}$. 


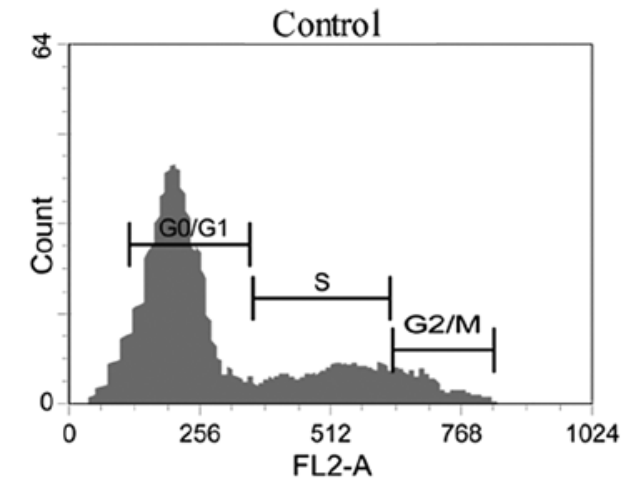

G0/G1 43.94 S 44.12 G2/M 11.94 apoptosis rate 2.93

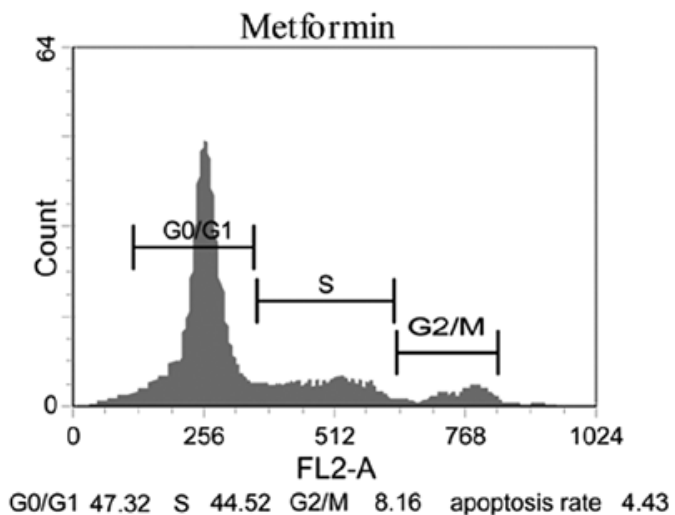

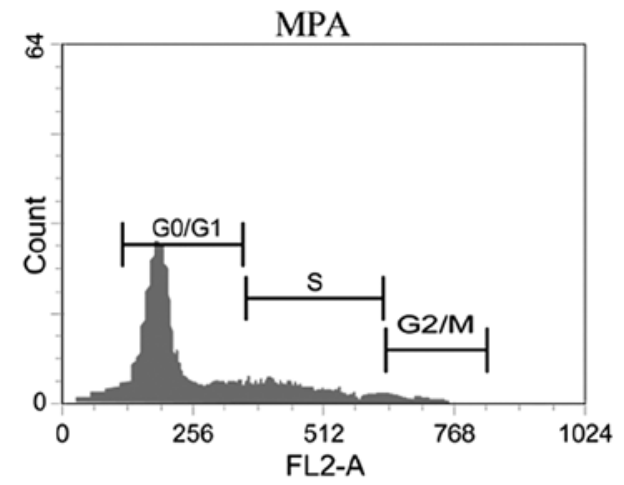

Go/G1 53.33 S $40.56 \quad$ G2/M 6.11 apoptosis rate 4.02

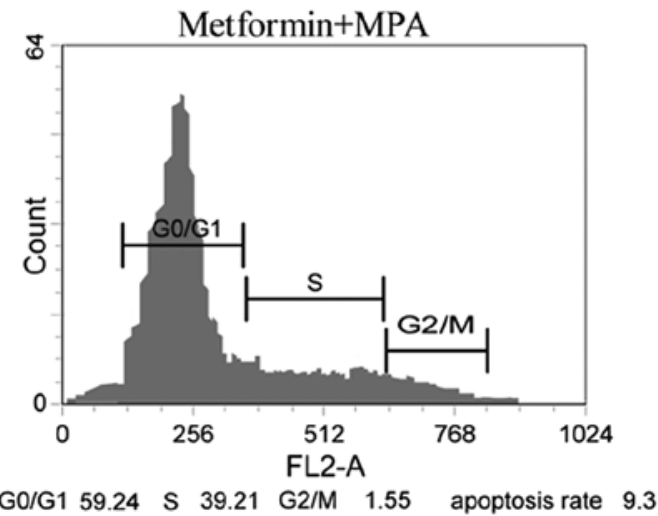

Figure 3. Metformin and (or) MPA induce cell cycle arrest in Ishikawa cells. Ishikawa cells were treated with PBS, metformin, MPA and metformin + MPA for $72 \mathrm{~h}$. Cells treated with PBS were considered as the control group. Metformin, MPA and metformin + MPA treatments all showed significantly stronger G0/G1 phase arresting effects compared with the control group. Furthermore, the G0/G1 phase arresting effect of metformin + MPA was significantly stronger than either of them used alone.
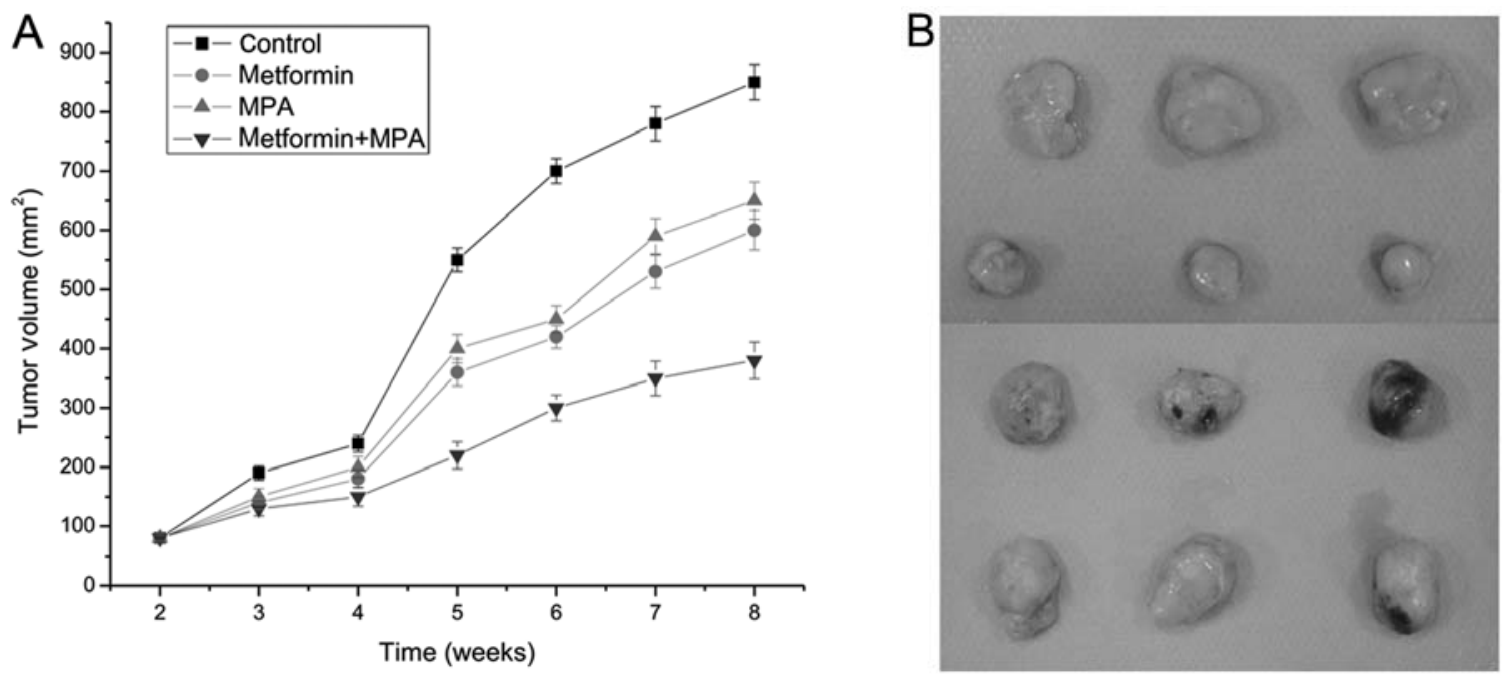

Control

Metformin+MPA

Metformin

MPA

Figure 4. (A) Xenograft tumor growth curve. (B) Effect of metformin and (or) MPA on xenograft tumors. Metformin, MPA and metformin + MPA all inhibited the growth of the xenograft tumors in a time-dependent manner. The combined use of metformin and MPA exhibited significant stronger inhibitory effect than either of the agents used alone. The results are presented as mean $\pm \mathrm{SE}$.

of metformin $(\mathrm{P}<0.001,<0.001$ and $<0.001$, respectively) or MPA ( $\mathrm{P}<0.001,<0.001$ and $<0.001$ respectively) used alone.

Effect of metformin and (or) MPA on the cell cycle. As displayed in Fig. 3, metformin, MPA and combined application of them all significantly inhibited the proliferation of the
Ishikawa cells. Compared with the control group, more cells were arrested in the G0/G1 phase, whereas, less cells were found in the G2/M phase when stimulated with metformin and (or) MPA. This synergistic arresting effect of the two agents was significantly stronger than that of metformin or MPA applied alone. 


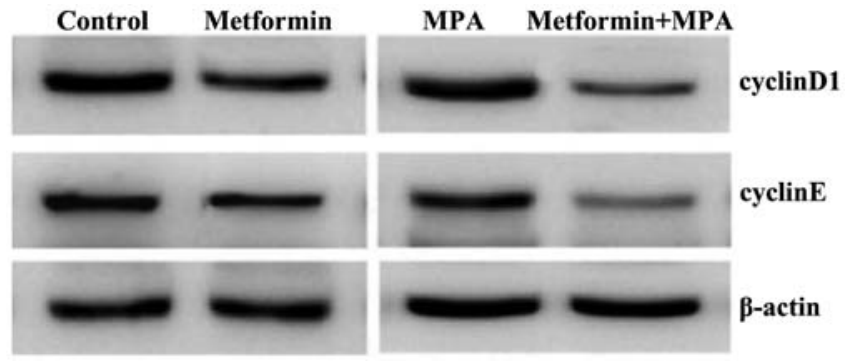

Figure 5. Effect of metformin and (or) MPA on the expression of cyclin D1 and cyclin E. Metformin and MPA treatment both significantly inhibited the expression of cyclin D1 and cyclin E. The inhibitory effect of metformin + MPA treatment was significantly stronger than that of metformin or MPA treatment alone.

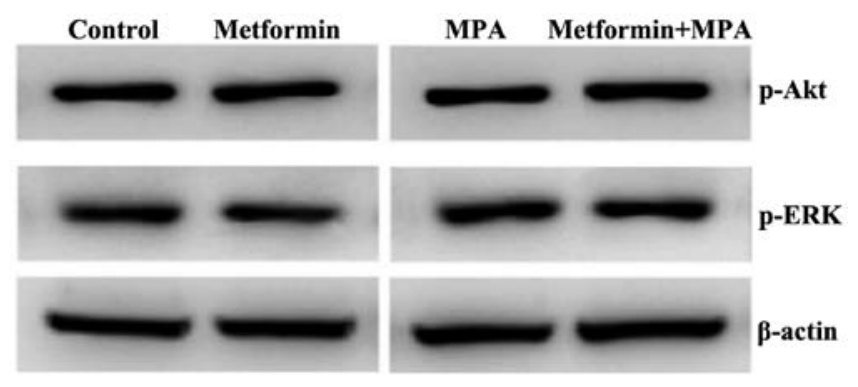

Figure 6. Effect of metformin and (or) MPA on PI3K/PKB and MAPK/ERK signaling pathways. There was no significant difference of the expression of p-Akt and p-ERK among the groups with different treatment.

Tumor growth assay in vivo. In this section, we evaluated the effect of metformin and (or) MPA on the growth of the xenograft tumor model. As displayed in Fig. 4, between the fifth to the eighth week, metformin $(\mathrm{P}=0.034,0.032,0.039$ and 0.030 , respectively), MPA $(\mathrm{P}=0.039,0.035,0.036$ and 0.033 respectively) and metformin + MPA $(\mathrm{P}=0.014,0.011,0.019$ and 0.018 , respectively) all significantly inhibited the growth of the xenograft tumors. Furthermore, between the fifth to the eighth week, the synergistic suppressive effect of metformin combined with MPA on tumor growth was significantly stronger than metformin $(\mathrm{P}=0.020,0.028,0.025$ and 0.024 , respectively) or MPA $(\mathrm{P}=0.017,0.021,0.019$ and 0.020 , respectively), alone. Although tumor growth appeared more severely suppressed by metformin than MPA (Fig. 4B), the difference was not statistically significant.

Western blot analysis of the xenograft tumors. Cyclin D1 and cyclin E play important roles in cell-cycle progression. The expression of these two proteins is reported as being positively associated with cell proliferation. The PI3K/Akt and MAPK/Ras signaling pathways are also significant factors in regulating cell proliferation and apoptosis. Therefore, the expression of Cyclin D1, cyclin E and phosphorylated Akt and ERK in xenograft tumors was evaluated. As displayed in Fig. 5, the expression of cyclin D1 and cyclin E was significantly decreased by metformin and (or) MPA compared with the control group. Although the inhibitory effect of metformin appeared stronger than that of MPA, the difference was not statistically significant. Furthermore, the combined effect of metformin and MPA was found significantly stronger than either of them used alone. Subsequently, we examined the phosphorylation of Akt and ERK in the xenograft tumors. Notably, as dipslayed in Fig. 6, we observed no difference in the key-protein phosphorylation among the groups with different treatments.

\section{Discussion}

The results provided by the present study demonstrated that both metformin and MPA have potent inhibitory effects on the development of EC cells. Furthermore, the synergistic effect of these two agents was significantly stronger than either of them used alone. Through arresting cancer cells in the G0/G1 phase, the agents promoted the apoptosis and inhibited the proliferation of the Ishikawa cells. Furthermore, these two agents potently inhibited the development of the xenograft tumors in vivo and the combined effect of them displayed greater inhibitory effect. The inhibition of the expression of cyclin D1 and cyclin E is probably one of the mechanisms of the synergistic effect of these two agents. Our findings indicated that the combined use of metformin and MPA may be a more effective strategy for the treatment of EC.

The anti-estrogen strategy had been applied in the treatment of EC for many years. MPA is one of the most popular agents clinically used in the treatment of EC. By binding to its receptor, especially the $\mathrm{B}$ subtype, progestin regulates multiple signaling pathways related to cell proliferation, apoptosis and differentiation (19). This inhibitory effect of MPA on cancer cells was revealed in a cell cycle phase-specific mode (20). The decreased expression and (or) inactivation of c-Myc, cyclin and the associated cyclin-dependent kinases (CDKs) appeared to play significant roles in the cell cycle-phase arresting effect (21-24). Cyclin is a family of proteins playing an important role in cell cycle progression. CDK family is also a key factor in cell cycle progression the activation of which is positively associated with the expression of cyclins. Enough cyclin binding to CDK is pivotal for cells to pass through the G1-phase. As indicated in our results, MPA effectively inhibited the proliferation of the Ishikawa cells in vitro and the G0/G1-phase arrest was probably one of the mechanisms. Subsequently, the animal study revealed that the MPA management significantly inhibited the growth of the xenograft tumors. The expression of cyclin D1 and cyclin E of the xenograft tumors treated with MPA was significantly lower than that of the controls. The results of the current study were similar to previous studies (25-27). Accordingly, it was indicated that MPA inhibits the development of EC through the cell cycle arrest.

Metformin is a common insulin-sensitizing agent used to treat type II diabetes. However, accumulating evidence indicated that metformin could be applied in the treatment of some malignant diseases including EC (28-30). Firstly, as an insulin-sensitizing agent, metformin decreased the circulating insulin levels by inhibiting the hepatic glucose and lipid synthesis, as well as by increasing muscle glucose uptake. As a result, the insulin induced proliferation-promoting and apoptosis-inhibiting effects were weakened by metformin. Sex hormone binding globulin (SHBG) is a significant serum sex hormone-concentration regulator which tightly binds to sex hormones. It was reported that insulin inhibited the 
production of SHBG (31) leading elevated free-estrogen levels to promote the development of EC. Since metformin downregulates serum insulin levels, circulating SHBG levels will be increased, resulting in less free-estrogen to stimulate the pathogenesis of EC. Secondly, insulin acts as an antitumor agent directly suppressing the development of EC. It is well-known that metformin phosphralytes LKB-1 and then AMP-activated protein kinase (AMPK) is activated which leads to the inactivation of mammalian target of rapamycin (mTOR)-signaling pathway (32). Furthermore, metformin was found to exert cell cycle-inhibitory effect on cancer cells by downregulating the expression of the related key proteins such as cyclin D1 and cyclin E (33-36). This cell cycle-arresting effect greatly impaired cell proliferation. These results were in line with that of the present study. In our study, metformin significantly inhibited the proliferation of the Ishikawa cells and arrested cancer cells in the G0/G1-phase. Furthermore, the growth of the xenograft tumors was significantly inhibited by metformin management. The expression of cyclin D1 and cyclin $\mathrm{E}$ were significantly lower. The data provided by the present study indicated that cell cycle phase-arrest can be used to explain the inhibitory effects of metformin on the development of EC.

To date, only a small number of studies have reported the combined application of metformin and progestin on the treatment of EC. It was revealed that metformin inhibited the expression of glyoxalase which is a key regulator of progestin-resistance to strengthen the therapeutic effect of progestin on EC cells (37). Furthermore, the inhibitory effect of metformin on the mTOR signaling pathway was associated with the upregulation of the expression of the progestin receptor in EC cells (38). These studies indicated that the combined application of metformin and progestin had stronger inhibitory effect on the EC cells than using either of the agents alone. However, the role played by metformin appeared adjuvant, but not synergistic. Furthermore, the common targets of the two agents were not provided. In the present study, the combined use of metformin and progestin exhibited stronger inhibitory effect on the development of EC than either of the two agents used alone. Furthermore, we revealed that cyclin D1 and cyclin E were the common targets of these two agents. The synergistic inhibitory effect of metformin and progestin on the expression of cyclin D1 and cyclin E caused cancer cell-arrest in the G0/G1 phase. As a result, the development of EC was severely delayed. In the present study, the expression of phosphorylated Akt and ERK was also evaluated. However, the differences were not significant among different groups. The data presented in the study indicated that G0/G1 phase-arrest induced by the downregulation of the expression of cyclin D1 and cyclin E is at least partly responsible for the synergistic effect of metformin and progestin on EC.

Although the present study provided interesting results, there are still some weak points. Firstly, only one dose of each agent was used in the present study, therefore it remains unknown whether the synergistic inhibitory effect is dose-dependent. Furthermore, the most optimal dose combination of the two agents was not provided. Secondly, since the observation time for the animal study was short, it is unknown whether there are any adverse impacts of the combination use of these two agents. Thirdly, only cyclin D1 and cyclin $\mathrm{E}$ were identified as the common targets of metformin and MPA, however the whole signaling network was not explored.

In conclusion, the present study provided novel insights into the treatment of EC. The combined application of metformin and MPA inhibited the development of EC in a synergistic manner. The downregulation of cyclin D1 and cyclin E was identified as one of the mechanisms of the synergistic inhibitory effect. Future studies are warranted to further evaluate the combined application of metformin and MPA and the mechanisms underlying this synergistic inhibitory effect.

\section{Acknowledgements}

The present study was generously supported by the Shandong Provincial Natural Science Foundation of China (no. ZR2016HL38).

\section{Competing interests}

The authors declare that they have no competing interests.

\section{References}

1. Key TJ and Pike MC: The dose-effect relationship between 'unopposed' oestrogens and endometrial mitotic rate: Its central role in explaining and predicting endometrial cancer risk. Br J Cancer 57: 205-212, 1988.

2. Henderson BE, Ross RK, Pike MC and Casagrande JT: Endogenous hormones as a major factor in human cancer. Cancer Res 42: 3232-3239, 1982.

3. Sjogren LL, Morch LS and Lokkegaard E: Hormone replacement therapy and the risk of endometrial cancer: A systematic review. Maturitas 91: 25-35, 2016.

4. Mu N, Zhu Y, Wang Y, Zhang H and Xue F: Insulin resistance: A significant risk factor of endometrial cancer. Gynecol Oncol 125: 751-757, 2012.

5. Berstein LM, Kvatchevskaya JO, Poroshina TE, Kovalenko IG, Tsyrlina EV, Zimarina TS, Ourmantcheeva AF, Ashrafian L and Thijssen JH: Insulin resistance, its consequences for the clinical course of the disease, and possibilities of correction in endometrial cancer.J Cancer Res Clin Oncol 130: 687-693, 2004.

6. Wang Y, Hua S, Tian W, Zhang L, Zhao J, Zhang H, Zhang W and Xue F: Mitogenic and anti-apoptotic effects of insulin in endometrial cancer are phosphatidylinositol 3-kinase/Akt dependent. Gynecol Oncol 125: 734-741, 2012.

7. Wang Y, Zhu Y, Zhang L, Tian W, Hua S, Zhao J, Zhang H and Xue F: Insulin promotes proliferation, survival, and invasion in endometrial carcinoma by activating the MEK/ERK pathway. Cancer Lett 322: 223-231, 2012.

8. Shao Y, Cheng S, Hou J, Zuo Y, Zheng W, Xia M and Mu N: Insulin is an important risk factor of endometrial cancer among premenopausal women: A case-control study in China. Tumour Biol 37: 4721-4726, 2016.

9. Hernandez AV, Pasupuleti V, Benites-Zapata VA, Thota P, Deshpande A and Perez-Lopez FR: Insulin resistance and endometrial cancer risk: A systematic review and meta-analysis. Eur J Cancer 51: 2747-2758, 2015.

10. Smith RA, Andrews KS, Brooks D, Fedewa SA, ManassaramBaptiste D, Saslow D, Brawley OW and Wender RC: Cancer screening in the United States, 2017: A review of current American Cancer Society guidelines and current issues in cancer screening. CA Cancer J Clin 67: 100-121, 2017.

11. Garrett A and Quinn MA: Hormonal therapies and gynaecological cancers. Best Pract Res Clin Obstet Gynaecol 22: 407-421, 2008.

12. Hawkes AL, Quinn M, Gebski V, Armes J, Brennan D and Janda M; feMME Trial Committee and Obermair A: Improving treatment for obese women with early stage cancer of the uterus: Rationale and design of the levonorgestrel intrauterine device \pm metformin \pm weight loss in endometrial cancer (feMME) trial. Contemp Clin Trials 39: 14-21, 2014. 
13. Nwanodi O: Progestin intrauterine devices and metformin Endometrial hyperplasia and early stage endometrial cancer medical management. Healthcare (Basel) 5: E30, 2017.

14. Umene K, Banno K, Kisu I, Yanokura M, Nogami Y, Tsuji K, Masuda K, Ueki A, Kobayashi Y, Yamagami W, et al: New candidate therapeutic agents for endometrial cancer: potential for clinical practice (review). Oncol Rep 29: 855-860, 2013.

15. Fournier A, Dossus L, Mesrine S, Vilier A, Boutron-Ruault MC, Clavel-Chapelon F and Chabbert-Buffet N: Risks of endometrial cancer associated with different hormone replacement therapies in the E3N cohort, 1992-2008. Am J Epidemiol 180: 508-517, 2014.

16. Decensi A, Puntoni M, Goodwin P, Cazzaniga M, Gennari A, Bonanni B and Gandini S: Metformin and cancer risk in diabetic patients: A systematic review and meta-analysis. Cancer Prev Res (Phila) 3: 1451-1461, 2010.

17. Mu N, Wang Y and Xue F: Metformin: A potential novel endometrial cancer therapy. Int J Gynecol Cancer 22: 181, 2012.

18. Workman P, Aboagye EO, Balkwill F, Balmain A, Bruder G, Chaplin DJ, Double JA, Everitt J, Farningham DA, Glennie MJ, et al: Guidelines for the welfare and use of animals in cancer research. $\mathrm{Br}$ J Cancer 102: 1555-1577, 2010.

19. Kim JJ and Chapman-Davis E: Role of progesterone in endometrial cancer. Semin Reprod Med 28: 81-90, 2010.

20. Musgrove EA and Sutherland RL: Cell cycle control by steroid hormones. Semin Cancer Biol 5: 381-389, 1994.

21. Musgrove EA, Lee CS, Cornish AL, Swarbrick A and Sutherland RL: Antiprogestin inhibition of cell cycle progression in T-47D breast cancer cells is accompanied by induction of the cyclin-dependent kinase inhibitor p21. Mol Endocrinol 11: 54-66, 1997.

22. Watts CK, Brady A, Sarcevic B, DeFazio A, Musgrove EA and Sutherland RL: Antiestrogen inhibition of cell cycle progression in breast cancer cells in associated with inhibition of cyclin-dependent kinase activity and decreased retinoblastoma protein phosphorylation. Mol Endocrinol 9: 1804-1813, 1995.

23. Musgrove EA, Swarbrick A, Lee CS, Cornish AL and Sutherland RL: Mechanisms of cyclin-dependent kinase inactivation by progestins. Mol Cell Biol 18: 1812-1825, 1998.

24. Yang S, Thiel KW and Leslie KK: Progesterone: The ultimate endometrial tumor suppressor. Trends Endocrinol Metab 22: 145-152, 2011.

25. Chang WT, Lin HL, Chang KL, Ker CG, Huang MC, Chen JS Kuo KK, Chen YL and Lee KT: Progesterone increases epirubicin's apoptotic effects in HepG2 cells by $S$ phase cell cycle arrest. Hepatogastroenterology 57: 107-113, 2010.

26. Hernández-Hernández OT, González-García TK and Camacho-Arroyo I: Progesterone receptor and SRC-1 participate in the regulation of VEGF, EGFR and Cyclin D1 expression in human astrocytoma cell lines. J Steroid Biochem Mol Biol 132: 127-134, 2012.
27. Rivas MA, Venturutti L, Huang YW, Schillaci R, Huang TH and Elizalde PV: Downregulation of the tumor-suppressor miR-16 via progestin-mediated oncogenic signaling contributes to breast cancer development. Breast Cancer Res 14: R77, 2012.

28. Soliman PT, Zhang Q, Broaddus RR, Westin SN, Iglesias D, Munsell MF, Schmandt R, Yates M, Ramondetta L and Lu KH: Prospective evaluation of the molecular effects of metformin on the endometrium in women with newly diagnosed endometrial cancer: A window of opportunity study. Gynecol Oncol 143: 466-471, 2016

29. Zou J, Hong L, Luo C, Li Z, Zhu Y, Huang T, Zhang Y, Yuan H, Hu Y, Wen T, et al: Metformin inhibits estrogen-dependent endometrial cancer cell growth by activating the AMPK-FOXO1 signal pathway. Cancer Sci 107: 1806-1817, 2016.

30. Gadducci A, Biglia N, Tana R, Cosio S and Gallo M: Metformin use and gynecological cancers: A novel treatment option emerging from drug repositioning. Crit Rev Oncol Hematol 105: 73-83, 2016.

31. Plymate SR, Matej LA, Jones RE and Friedl KE: Inhibition of sex hormone-binding globulin production in the human hepatoma (Hep G2) cell line by insulin and prolactin. J Clin Endocrinol Metab 67: 460-464, 1988.

32. Kourelis TV and Siegel RD: Metformin and cancer: New applications for an old drug. Med Oncol 29: 1314-1327, 2012.

33. Scherbakov AM, Sorokin DV, Tatarskiy VJ Jr, Prokhorov NS, Semina SE, Berstein LM and Krasil'nikov MA: The phenomenon of acquired resistance to metformin in breast cancer cells: The interaction of growth pathways and estrogen receptor signaling. IUBMB Life 68: 281-292, 2016.

34. Cai X, Hu X, Tan X, Cheng W, Wang Q, Chen X, Guan Y, Chen C and Jing X: Metformin induced AMPK activation, G0/G1 phase cell cycle arrest and the inhibition of growth of esophageal squamous cell carcinomas in vitro and in vivo. PLoS One 10: e133349, 2015.

35. Sikka A, Kaur M, Agarwal C, Deep G and Agarwal R: Metformin suppresses growth of human head and neck squamous cell carcinoma via global inhibition of protein translation. Cell Cycle 11: 1374-1382, 2012

36. Tosca L, Ramé C, Chabrolle C, Tesseraud S and Dupont J: Metformin decreases IGF1-induced cell proliferation and protein synthesis through AMP-activated protein kinase in cultured bovine granulosa cells. Reproduction 139: 409-418, 2010.

37. Zhang Z, Dong L, Sui L, Yang Y, Liu X, Yu Y, Zhu Y and Feng Y: Metformin reverses progestin resistance in endometrial cancer cells by downregulating GloI expression. Int J Gynecol Cancer 21: 213-221, 2011.

38. Xie Y, Wang YL, Yu L, Hu Q, Ji L, Zhang Y and Liao QP: Metformin promotes progesterone receptor expression via inhibition of mammalian target of rapamycin (mTOR) in endometrial cancer cells. J Steroid Biochem Mol Biol 126: 113-120, 2011. 